\title{
Developing Buoyancy Driven Flow of a Nanofluid in a Vertical Channel Subject to Heat Flux
}

\author{
Nirmal C. Sacheti, ${ }^{1}$ Pallath Chandran, ${ }^{1}$ Ashok K. Singh, ${ }^{2}$ and Beer S. Bhadauria ${ }^{2,3}$ \\ ${ }^{1}$ Department of Mathematics \& Statistics, College of Science, Sultan Qaboos University, Al Khod, 123 Muscat, Oman \\ ${ }^{2}$ Department of Mathematics, Banaras Hindu University, Varanasi 221005, India \\ ${ }^{3}$ Department of Applied Mathematics, School of Physical Sciences, Babasaheb Bhimrao Ambedkar University, Lucknow 226025, India
}

Correspondence should be addressed to Pallath Chandran; chandran@squ.edu.om

Received 24 August 2013; Accepted 17 November 2013; Published 2 February 2014

Academic Editor: Alberto Cardona

Copyright (C) 2014 Nirmal C. Sacheti et al. This is an open access article distributed under the Creative Commons Attribution License, which permits unrestricted use, distribution, and reproduction in any medium, provided the original work is properly cited.

\begin{abstract}
The developing natural convective flow of a nanofluid in an infinite vertical channel with impermeable bounding walls has been investigated. It is assumed that the nanofluid is dominated by two specific slip mechanisms and that the channel walls are subject to constant heat flux and isothermal temperature, respectively. The governing nonlinear partial differential equations coupling different transport processes have been solved numerically. The variations of velocity, temperature, and nanoparticles concentration have been discussed in relation to a number of physical parameters. It is seen that the approach to the steady-state profiles of velocity and temperature in the present work is different from the ones reported in a previous study corresponding to isothermal wall conditions.
\end{abstract}

\section{Introduction}

Analytical and numerical studies of natural convective processes involving viscous fluids have been the subject of comprehensive investigations during the last five decades or so, primarily stemming from their wide-ranging applications in several disciplines of engineering and technology. The scope and extent of such investigations have been enormous because of the sheer large number of aspects covered: steady or unsteady flows, permeable or impermeable boundaries, electrically conducting or nonconducting fluids, presence or absence of applied magnetic field, finite or infinite flow domain, adiabatic or isothermal boundary conditions, and two- or three-dimensional nature of flow, to name a few. In particular, a large body of literature exists on the transient free convective flows of viscous incompressible fluids through infinite vertical channels, subject to a variety of imposed conditions on the bounding walls (see, for example, [1-3]).

In recent years, an increasing number of theoretical as well as experimental investigations related to natural convection in nanofluids have drawn the attention of researchers because of the potentially large variety of real life applications in engineering and industry. As is known, nanofluids are engineered colloids of nanoparticles having diameters of the order 1-100 nanometers (nm) and a base fluid having very high thermal conductivity as well as high heat transfer coefficients [4-7]. They are mixtures of base fluids-for example, water or ethylene-glycol-pervaded by a very small amount of nanoparticles such as metallic or metallic oxide particles $\left(\mathrm{Cu}, \mathrm{CuO}, \mathrm{Al}_{2} \mathrm{O}_{3}\right)$. Such fluids are thus considered suspensions. However, their particle size is so small that they approach the size of fluid particles. Because of this, nanofluids can be considered as single-phase fluids instead of two-phase. It has also been treated in the literature as a Newtonian fluid with a constant viscosity, in a number of applications. Also, nanofluids find a large number of industrial applications such as power manufacturing, transportation, cooling of highenergy electronic devices, nuclear systems, and nanodrugs, among others. Due to such wide-ranging applications, a vast body of literature related to nanofluids can be found in the recent literature, starting from mid-nineties [8-20]. Most of these works are devoted to the study of free, forced, or mixed convection in nanofluids in the presence or absence of porous media. 
In the investigations of convective flows involving solid boundaries, the thermal conditions at the bounding surfaces are known to play key roles in the developing boundary layer flows. In a very recent work, Sacheti et al. [21] carried out a numerical study dealing with transient two-dimensional free convection in a nanofluid through a vertical channel with rigid walls. Assuming the channel walls to be isothermaleither heated or cooled-the authors discussed developing profiles of velocity, temperature, and nanoparticle concentration. The effects of a number of nondimensional variables were clearly brought out in this study, showcasing various transport mechanisms. The present work extends our earlier analysis [21] by considering the natural convection in a nanofluid dominated by two key slip mechanisms: Brownian diffusion and thermophoresis. It is assumed that one of the boundaries is subjected to heat flux. We investigate the transient nature of the flow when the heat is supplied at the constant rate at the left channel wall while the other wall is isothermal. The coupled nonlinear parabolic partial differential equations governing the flow subject to the relevant initial and boundary conditions have been solved by an implicit finite-difference scheme. The effects of the relevant transport phenomena characterized by Lewis number, buoyancy ratio parameter, Brownian motion parameter, and thermophoresis parameter have been analyzed. Furthermore, the temporal progression of the field variables-velocity, temperature, and nanoparticle volume fraction-from the unsteady state to the steady state has been examined. We have compared the results of our study involving heat flux wall condition at a boundary with the corresponding results for the isothermal wall conditions [21]. It has been observed that the approach to the steady-state values of fluid velocity and temperature follow different patterns in these two thermal cases.

\section{Governing Equations}

We consider a transient free convective flow between two vertical infinite walls situated at $y^{\prime}=0$ and $y^{\prime}=L$. Each wall is assumed to be impermeable and perfectly thermally conducting. The coordinate system is chosen such that the $x^{\prime}-$ axis measures the distance along the left wall and the $y^{\prime}$-axis measures the distance normal to it. Initially, the temperatures and volumetric fractions of nanoparticles as well as the walls are the same and are taken to be $T_{c}^{\prime}$ and $\phi_{c}^{\prime}$, respectively. At the time $t^{\prime}>0$, heat is supplied at a constant rate at the inner surface of the left wall while the inner surface of the right wall $\left(y^{\prime}=L\right)$ is maintained at a constant temperature $T_{c}^{\prime}$ which causes free convection currents in the channel. In addition, the volumetric fraction of nanoparticles is assumed to vary from $\phi_{h}^{\prime}$ on the inner surface of the left wall to $\phi_{c}^{\prime}$ on the inner surface of the right wall. Also, the Boussinesq approximation is assumed. The conservation equations of momentum, energy, and nanoparticles volume fraction are $[8,14,15,21]$

$$
\begin{aligned}
\rho_{f} \frac{\partial u^{\prime}}{\partial t^{\prime}}=\mu \frac{\partial^{2} u^{\prime}}{\partial y^{\prime 2}}+ & {\left[\left(1-\phi_{c}^{\prime}\right) \rho_{f} \beta\left(T^{\prime}-T_{c}^{\prime}\right)\right.} \\
& \left.-\left(\rho_{p}-\rho_{f}\right)\left(\phi^{\prime}-\phi_{c}^{\prime}\right)\right] g,
\end{aligned}
$$

$$
\begin{aligned}
&(\rho c)_{f} \frac{\partial T^{\prime}}{\partial t^{\prime}}= k \frac{\partial^{2} T^{\prime}}{\partial y^{\prime 2}} \\
&+(\rho c)_{p}\left[D_{B} \frac{\partial \phi^{\prime}}{\partial y^{\prime}} \frac{\partial T^{\prime}}{\partial y^{\prime}}+\left(\frac{D_{T}}{T_{c}^{\prime}}\right)\left(\frac{\partial T^{\prime}}{\partial y^{\prime}}\right)^{2}\right] \\
& \frac{\partial \phi^{\prime}}{\partial t^{\prime}}=D_{B} \frac{\partial^{2} \phi^{\prime}}{\partial y^{\prime 2}}+\left(\frac{D_{T}}{T_{c}^{\prime}}\right) \frac{\partial^{2} T^{\prime}}{\partial y^{\prime 2}} .
\end{aligned}
$$

Here $u^{\prime}$ is the velocity component of the fluid in the $x^{\prime}$-direction, $T^{\prime}$ is the temperature of nanofluid, $\phi^{\prime}$ is the nanoparticle volume fraction, $g$ is the acceleration due to the gravity, $\beta$ is the coefficient of thermal expansion, $\rho_{f}$ is the density of the fluid, $\mu$ is the viscosity of the fluid assumed to be constant, $\rho_{p}$ is the density of the particles, $(\rho c)_{f}$ is the heat capacity of the fluid, $k$ is the thermal conductivity of the fluid, $q$ is the constant heat flux on the left wall, and $(\rho c)_{p}$ is the effective heat capacity of the particles. Further, $D_{B}$ and $D_{T}$ are the coefficients of the Brownian diffusion and the thermophoresis diffusion, respectively.

Associated initial and boundary conditions on the velocity, temperature, and nanoparticle volume fraction are given, respectively, by

$$
\begin{aligned}
& t^{\prime} \leq 0: u^{\prime}=0, \quad T^{\prime}=T_{c}^{\prime}, \quad \phi^{\prime}=\phi_{c}^{\prime} \quad \text { for } 0 \leq y^{\prime} \leq L, \\
& t^{\prime}>0: u^{\prime}=0, \quad q=-k \frac{\partial T^{\prime}}{\partial y^{\prime}}, \quad \phi^{\prime}=\phi_{h}^{\prime} \quad \text { at } y^{\prime}=0, \\
& u^{\prime}=0, \quad T^{\prime}=T_{c}^{\prime}, \quad \phi^{\prime}=\phi_{c}^{\prime} \quad \text { at } y^{\prime}=L .
\end{aligned}
$$

We now introduce nondimensional variables:

$$
\begin{gathered}
y=\frac{y^{\prime}}{L}, \quad t=\frac{t^{\prime} \alpha}{L^{2}}, \quad u=\frac{u^{\prime} \alpha}{\left\{\beta g L^{2}\left(1-\phi_{c}^{\prime}\right)(q L / k)\right\}}, \\
T=\frac{\left(T^{\prime}-T_{c}^{\prime}\right)}{(q L / k)}, \quad \phi=\frac{\left(\phi^{\prime}-\phi_{c}^{\prime}\right)}{\left(\phi_{h}^{\prime}-\phi_{c}^{\prime}\right)} .
\end{gathered}
$$

In view of (3), the conservation equations (1) transform to

$$
\begin{gathered}
\frac{\partial u}{\partial t}=\operatorname{Pr} \frac{\partial^{2} u}{\partial y^{2}}+T-N \phi \\
\frac{\partial T}{\partial t}=\frac{\partial^{2} T}{\partial y^{2}}+\left(n_{b}\right) \frac{\partial \phi}{\partial y} \frac{\partial T}{\partial y}+\left(n_{t}\right)\left(\frac{\partial T}{\partial y}\right)^{2}, \\
\frac{\partial \phi}{\partial t}=\frac{1}{\operatorname{Le}} \frac{\partial^{2} \phi}{\partial y^{2}}+\frac{\left(n_{t}\right)}{(\mathrm{Le})\left(n_{b}\right)} \frac{\partial^{2} T}{\partial y^{2}},
\end{gathered}
$$

where $\alpha=k /(\rho c)_{f}$. The initial and boundary conditions transform to

$$
\begin{aligned}
& t \leq 0: u=0, T=0, \quad \phi=0 \quad \text { for } 0 \leq y \leq 1 ; \\
& t>0: u=0, \quad \frac{\partial T}{\partial y}=-1, \quad \phi=1 \quad \text { at } y=0 ; \\
& u=0, \quad T=0, \quad \phi=0 \quad \text { at } y=1 .
\end{aligned}
$$


The nondimensional physical parameters appearing in the above equations are the Prandtl number Pr, the Lewis number Le and the buoyancy ratio parameter $N$. The remaining parameters $n_{b}$ and $n_{t}$ are, respectively, the Brownian motion parameter and the thermophoresis parameter. The nondimensional parameters appearing in (4) are defined by

$$
\begin{gathered}
\operatorname{Pr}=\frac{\mu}{\rho_{f} \alpha}, \quad \operatorname{Le}=\frac{\alpha}{D_{B}}, \quad N=\frac{k\left(\rho_{p}-\rho_{f}\right)\left(\phi_{h}^{\prime}-\phi_{c}^{\prime}\right)}{\left(1-\phi_{c}^{\prime}\right) q L \beta \rho_{f}}, \\
n_{b}=\frac{D_{B}(\rho c)_{p}}{\alpha(\rho c)_{f}}\left(\phi_{h}^{\prime}-\phi_{c}^{\prime}\right), \quad n_{t}=\frac{D_{T}(\rho c)_{p} q L}{k \alpha(\rho c)_{f} T_{c}^{\prime}} .
\end{gathered}
$$

\section{Results and Discussion}

In order to solve the initial-boundary-value problem described by (4) and (5), we have used an implicit finitedifference method for the variables $u, T$, and $\phi$, as functions of $t$ and $y$. Using the central difference scheme for the space derivative and the backward difference scheme for the time derivative, the obtained finite difference equations have been reduced to a tridiagonal matrix which, in turn, has been solved by the Thomas algorithm. At the end of each time step, the sequence of function evaluations followed $\phi \rightarrow T \rightarrow u$ in that order. The unsteady values of the physical variables corresponding to a specific instant have been obtained by iterations on the nondimensional temporal variable. The computation is then advanced until a steady state is reached for each of the three physical variables. For the computation, we had chosen 21 grid points for the space variable while the time step was initially taken equal to 0.0004 . In order to ensure the stability and convergence of the implicit finite difference method, the computations were repeated by taking smaller values of time step such as 0.0003 and 0.0002 . In these cases, however, there were no significant changes in the results which confirms the stability and convergence of the implicit finite difference method used.

The results presented in this section correspond to the Prandtl number ( $\mathrm{Pr}$ ) equal to 7 . In the following, we will focus our attention on discussing the effects of the physical parameters-Lewis number (Le), Brownian motion parameter $\left(n_{b}\right)$, thermophoresis parameter $\left(n_{t}\right)$, and buoyancy ratio parameter $(N)$-on the field variables. Before we begin our analyses of various features observed in Figures 1-10, we wish to draw the attention of the readers to the fact that these parameters-Le, $n_{b}, n_{t}$, and $N$-have specific physical relevance. They arise either from the nanofluid model in this study or the imposed wall conditions related to a nanofluid variable. For instance, the Lewis number incorporates the relative influence of thermal diffusion and the Brownian diffusion. The latter is one of the key slip mechanisms associated with the nanofluid. The parameter $n_{b}$, on the other hand, describes the combined influence of the Brownian diffusion and the imposed unequal wall conditions for the nanoparticle volume fraction. The parameter $n_{t}$ is directly related to the thermophoresis which is yet another slip mechanism. The parameter $N$, the buoyancy parameter, arises due to

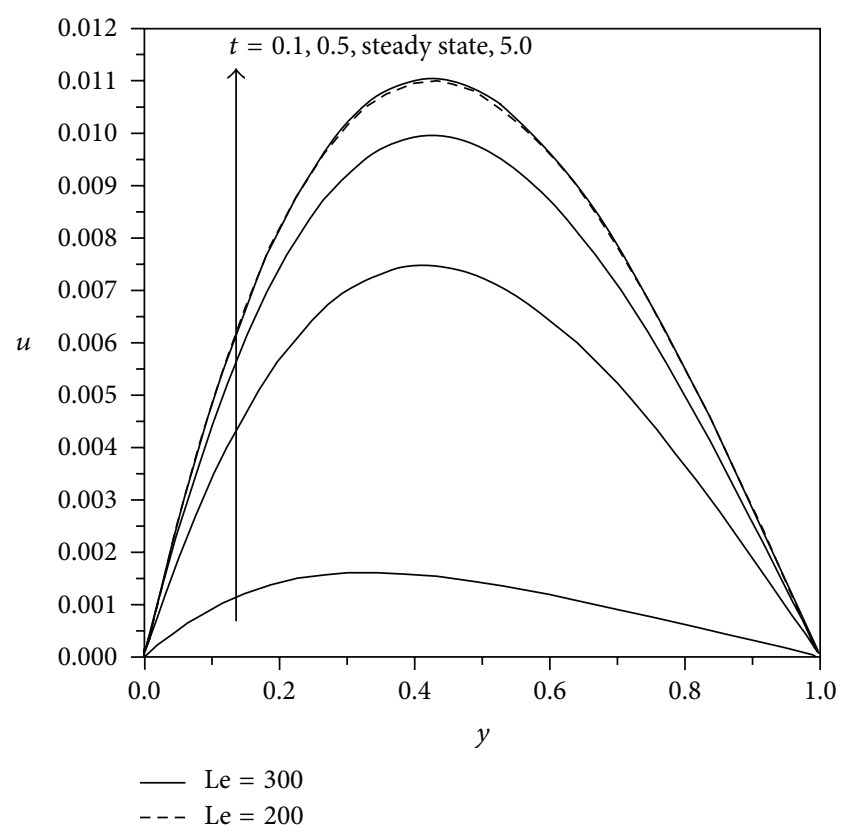

FIGURE 1: Variations of $u$ with respect to $y$ for $N=0.1, n_{b}=0.1$, and $n_{t}=0.01$.

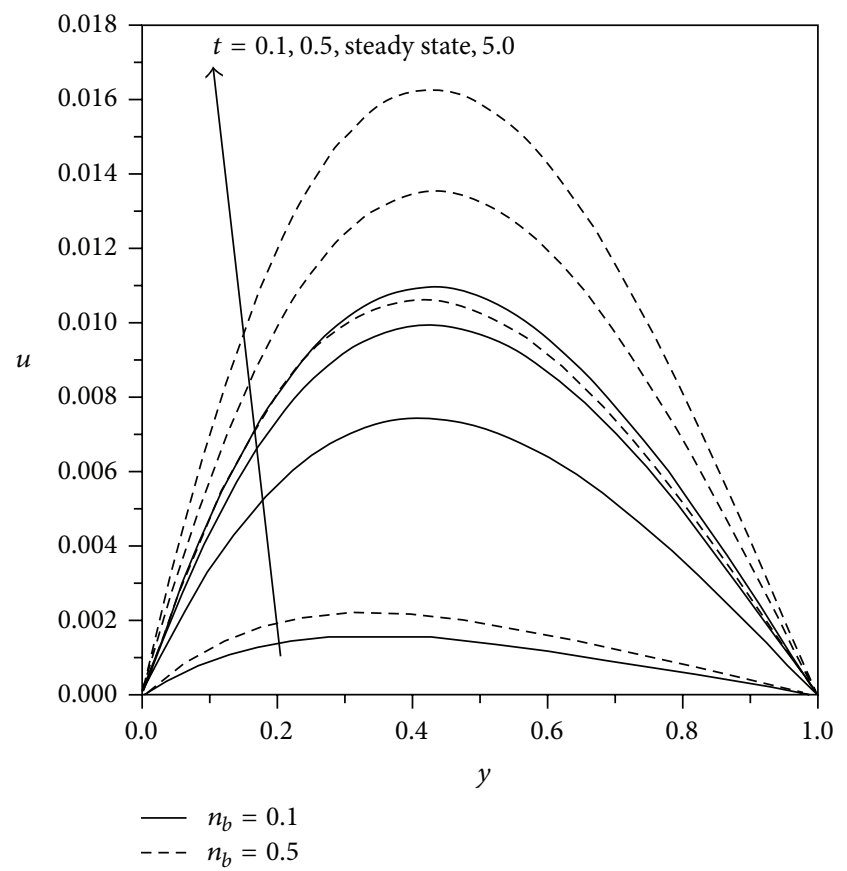

FIgURE 2: Variations of $u$ with respect to $y$ for $N=0.1, n_{t}=0.01$, and $\mathrm{Le}=200$

differentials in the densities as well as imposed nanoparticle volume fractions.

In Figures 1-4, we have shown the effects of each of the above four parameters on the developing fluid velocity. The velocity profiles, as expected, tend to become parabolic with time. However, the profiles are not symmetric due to the interplay between the buoyancy forces and the nanofluid 
TABLE 1: Skin friction, left-wall temperature, and Sherwood number $(\operatorname{Pr}=7)$.

\begin{tabular}{|c|c|c|c|c|c|c|c|}
\hline Le & $N$ & $n_{b}$ & $n_{t}$ & $t$ & $\tau$ & $T(0, t)$ & Sh \\
\hline \multirow{4}{*}{200} & \multirow{4}{*}{0.1} & \multirow{4}{*}{0.1} & \multirow{4}{*}{0.01} & 0.1 & 0.0119 & 0.4267 & 29.213 \\
\hline & & & & 0.5 & 0.0399 & 0.9175 & 12.459 \\
\hline & & & & 5.0 & 0.0557 & 1.2008 & 3.5761 \\
\hline & & & & 147 (SS) & 0.0514 & 1.1632 & 1.0086 \\
\hline \multirow{4}{*}{200} & \multirow{4}{*}{0.1} & \multirow{4}{*}{0.1} & \multirow{4}{*}{0.1} & 0.1 & 0.0218 & 0.7742 & 29.155 \\
\hline & & & & 0.5 & 0.0764 & 1.7220 & 12.441 \\
\hline & & & & 5.0 & 0.1180 & 2.4249 & 3.7211 \\
\hline & & & & 147 (SS) & 0.1128 & 2.3614 & 1.3778 \\
\hline \multirow{4}{*}{200} & \multirow{4}{*}{0.1} & \multirow{4}{*}{0.5} & \multirow{4}{*}{0.01} & 0.1 & 0.0165 & 0.5786 & 29.218 \\
\hline & & & & 0.5 & 0.0572 & 1.2831 & 12.466 \\
\hline & & & & 5.0 & 0.0822 & 1.6817 & 3.5783 \\
\hline & & & & 147 (SS) & 0.0680 & 1.4364 & 1.0091 \\
\hline \multirow{4}{*}{200} & \multirow{4}{*}{0.5} & \multirow{4}{*}{0.1} & \multirow{4}{*}{0.01} & 0.1 & 0.0109 & 0.4267 & 29.213 \\
\hline & & & & 0.5 & 0.0369 & 0.9175 & 12.459 \\
\hline & & & & 5.0 & 0.0470 & 1.2008 & 3.5761 \\
\hline & & & & 147 (SS) & 0.0324 & 1.1632 & 1.0086 \\
\hline \multirow{4}{*}{300} & \multirow{4}{*}{0.1} & \multirow{4}{*}{0.1} & \multirow{4}{*}{0.01} & 0.1 & 0.0119 & 0.4270 & 32.639 \\
\hline & & & & 0.5 & 0.0400 & 0.9185 & 16.253 \\
\hline & & & & 5.0 & 0.0562 & 1.2043 & 4.3805 \\
\hline & & & & 210 (SS) & 0.0514 & 1.1633 & 1.0123 \\
\hline
\end{tabular}

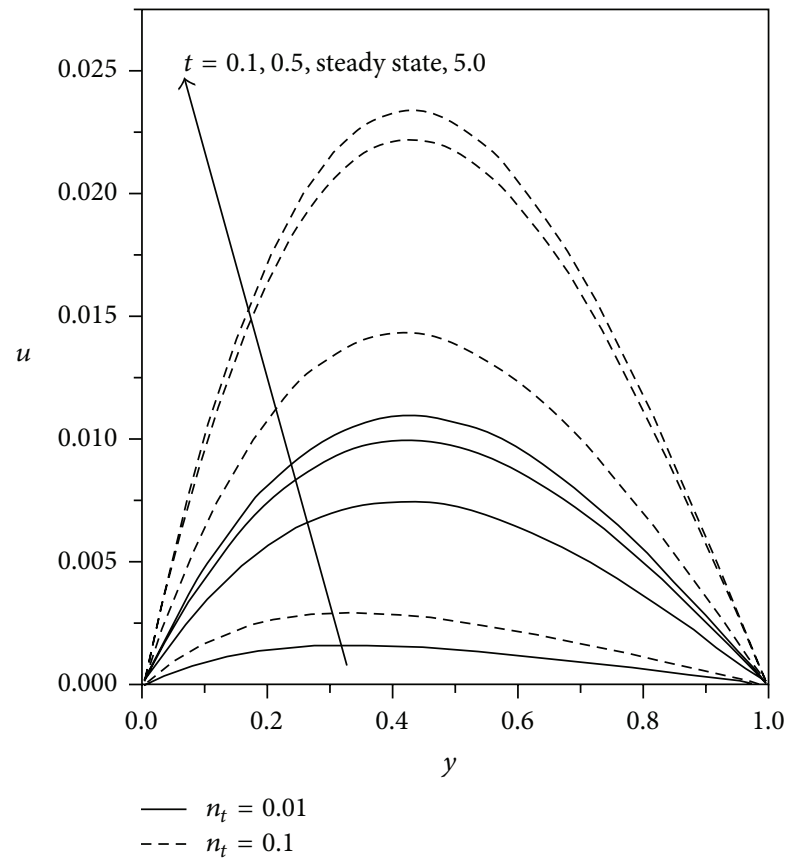

FIGURE 3: Variations of $u$ with respect to $y$ for $N=0.1, n_{b}=0.1$, and $\mathrm{Le}=200$.

characteristics. From Figure 1, we observe that the effect of the Lewis number on the velocity is almost negligible. As regards the effect of the Brownian motion parameter $n_{b}$-with the remaining three parameters fixed-we notice, from Figure 2, that the fluid velocity increases appreciably with increasing $n_{b}$. From these two figures, one may thus note that the velocity

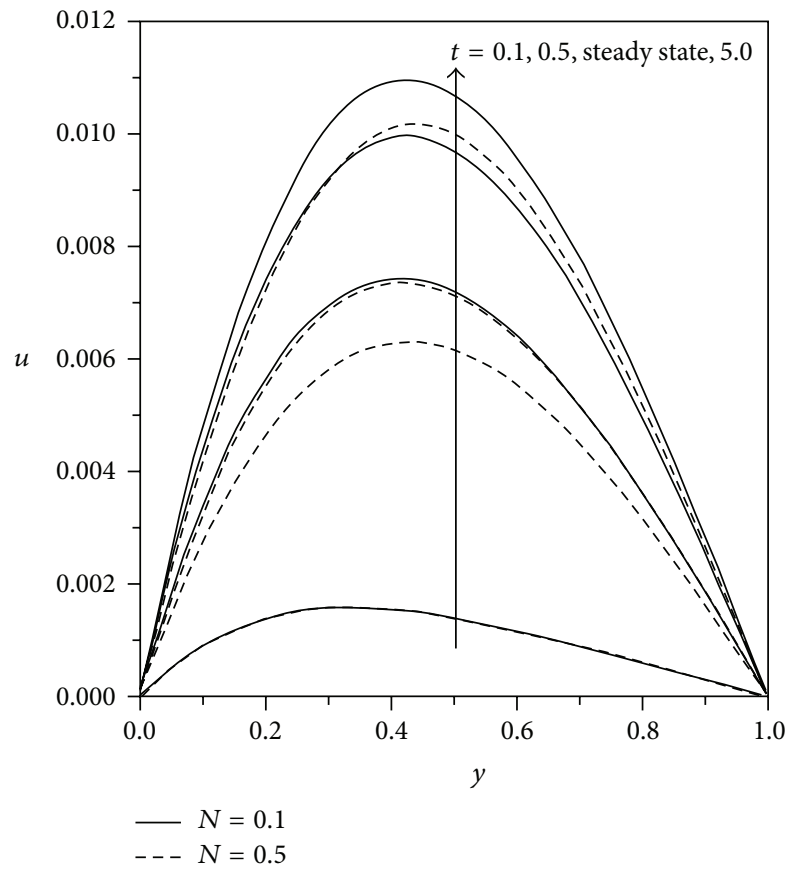

FIGURE 4: Variations of $u$ with respect to $y$ for $n_{b}=0.1, n_{t}=0.01$, and $\mathrm{Le}=200$.

profiles are sensitive more with respect to the imposed particle volume fraction differentials than the Brownian diffusion. As regards the influence of the thermophoresis parameter $n_{t}$ on the velocity (see Figure 3 ), we observe that the effect is similar to that of the parameter $n_{b}$. On the other hand, as can be seen from Figure 4, the nanofluid velocity in 


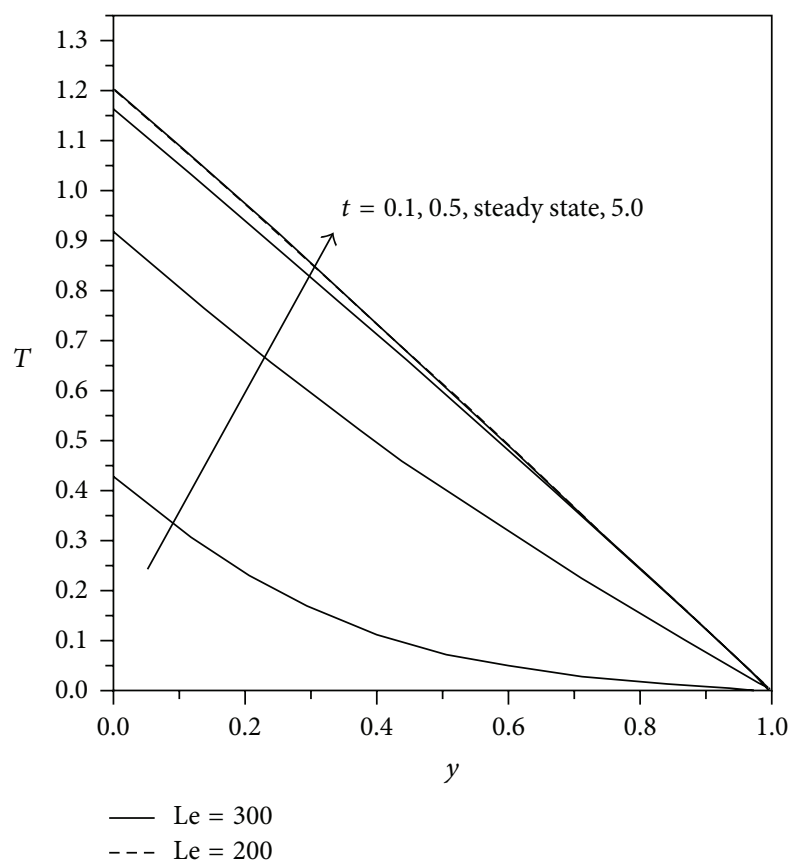

FIgURE 5: Variations of $T$ with respect to $y$ for $N=0.1, n_{b}=0.1$, and $n_{t}=0.01$.

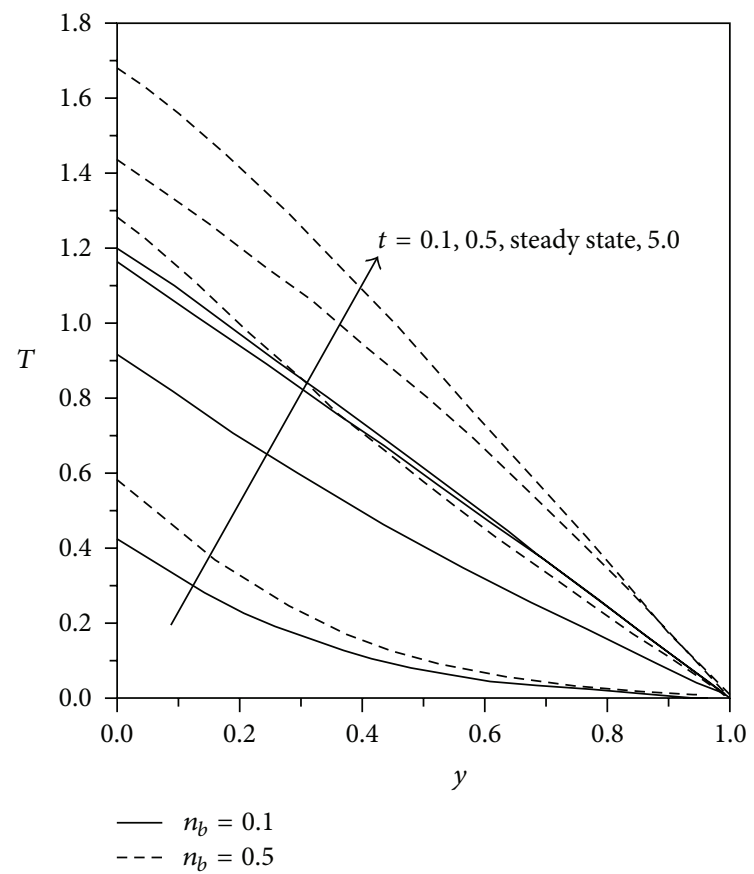

FIgURE 6: Variations of $T$ with respect to $y$ for $N=0.1, n_{t}=0.01$, and $\mathrm{Le}=200$.

the channel decreases with the enhancement of the buoyancy ratio parameter $N$. A common but interesting feature in all four figures is worth noting: while the transient velocity increases with time $t$ at all points of the channel, the steadystate values show a bit of reversal from the peak values. This steady-state behavior is different to the case when the two

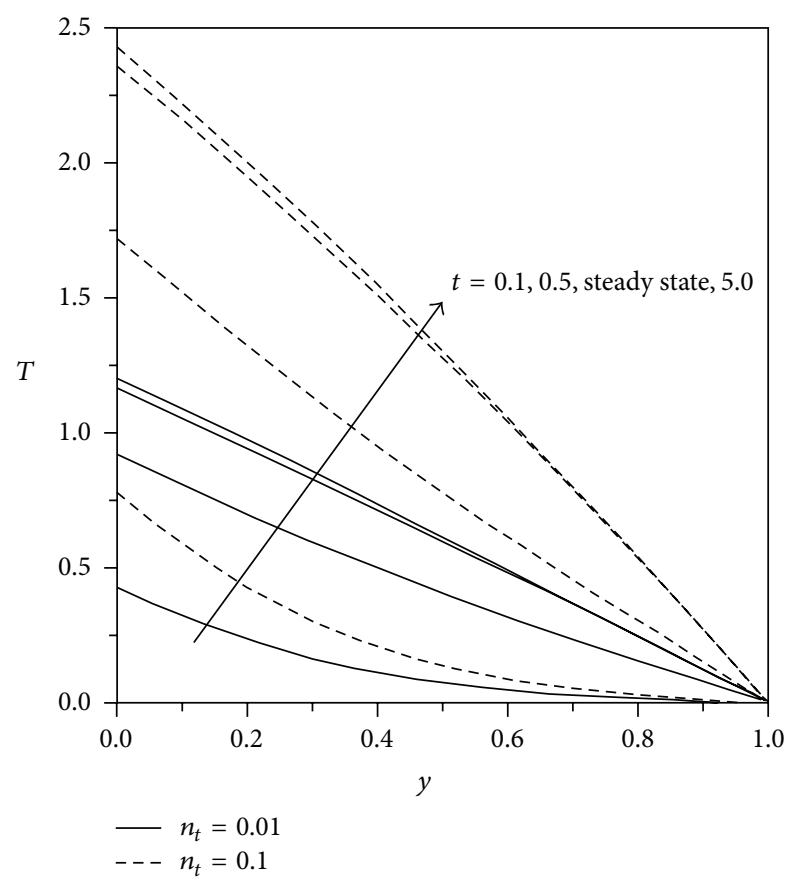

FIgURE 7: Variations of $T$ with respect to $y$ for $N=0.1, n_{b}=0.1$, and $\mathrm{Le}=200$.

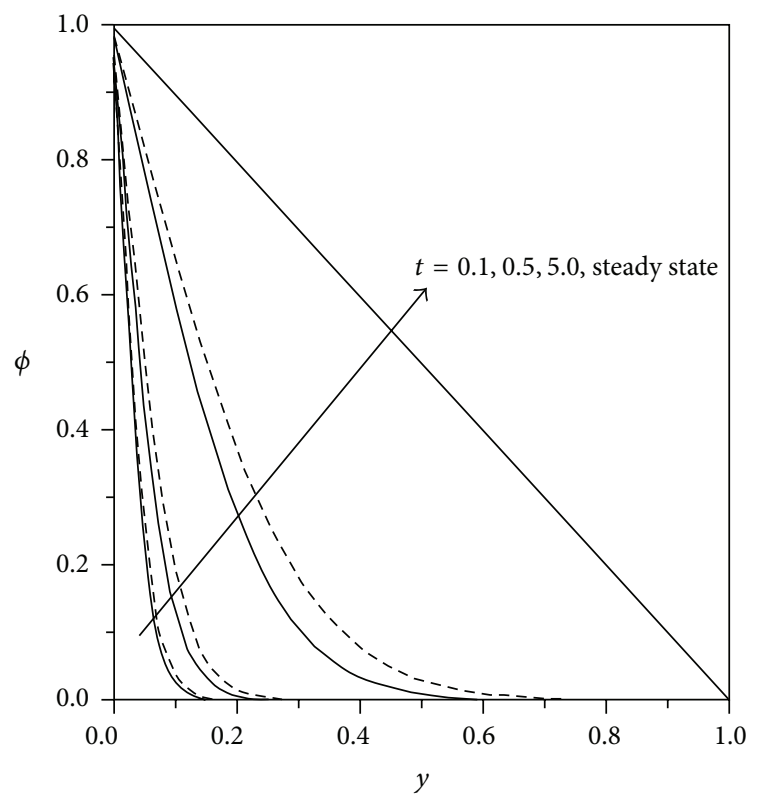

$-\mathrm{Le}=300$

FIGURE 8: Variations of $\phi$ with respect to $y$ for $N=0.1, n_{b}=0.1$, and $n_{t}=0.01$.

walls of the channel are isothermal [21]. In the latter case, it was seen that the transition from the unsteady to the steady state followed a monotonically increasing pattern.

In Figures 5-7, we have shown the variation of nanofluid temperature $T$ with respect to the parameters Le, $n_{b}$, and $n_{t}$, respectively. From Figure 5, we observe that the Lewis 


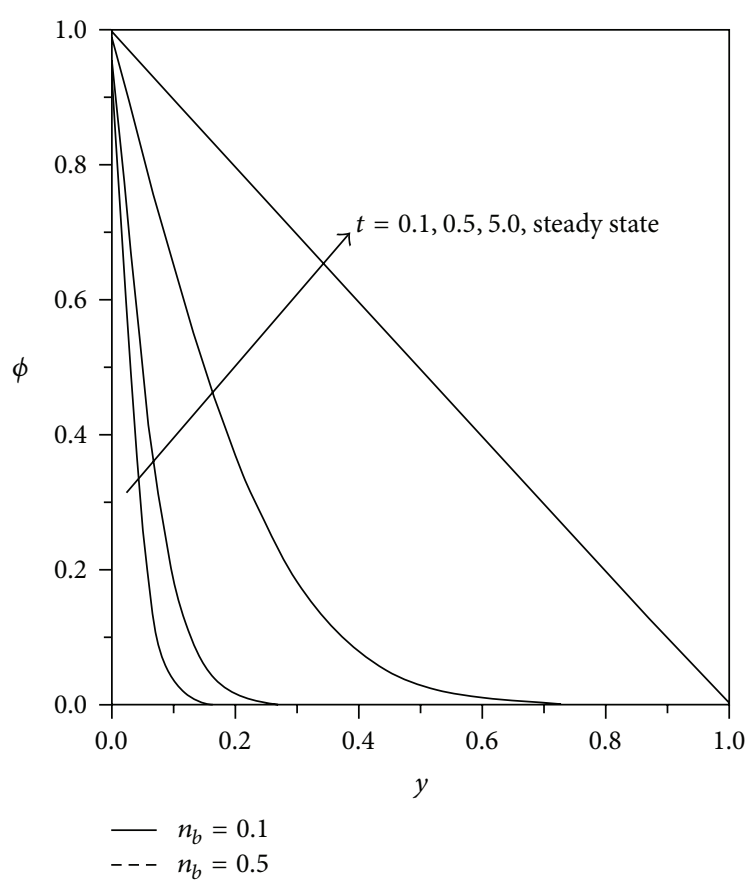

Figure 9: Variations of $\phi$ with respect to $y$ for $N=0.1, n_{t}=0.01$, and $\mathrm{Le}=200$.

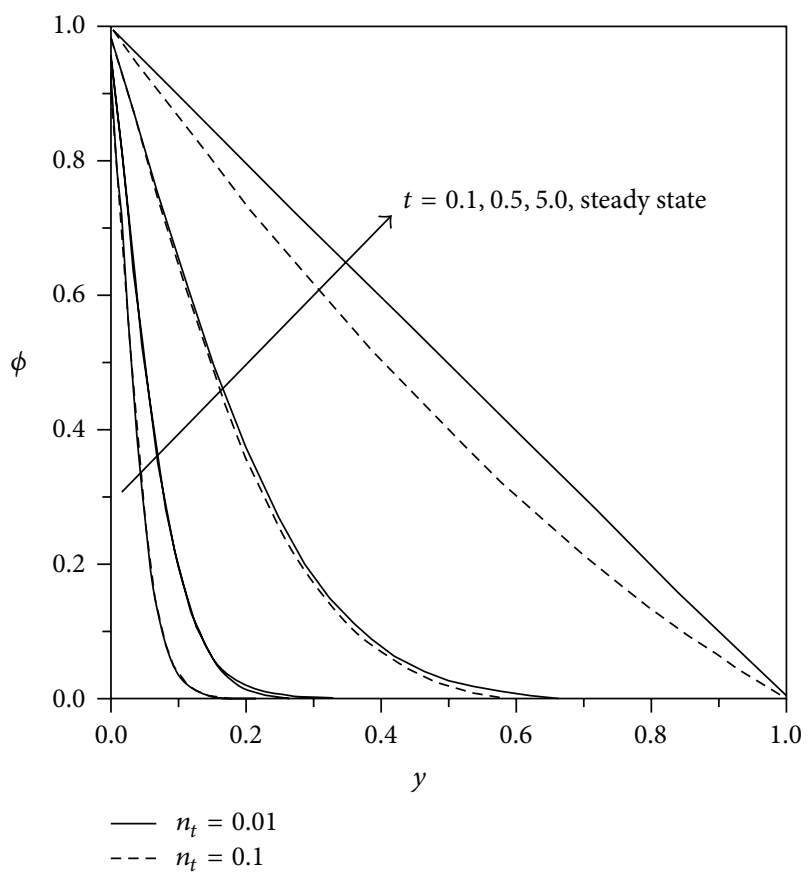

FIGURE 10: Variations of $\phi$ with respect to $y$ for $N=0.1, n_{b}=0.1$, and $\mathrm{Le}=200$.

number has negligible effect on the temperature. However, Brownian motion and thermophoresis of the nanoparticles tend to increase the temperature in the channel (Figures 6 and 7). The temporal behavior of the temperature is similar to the one observed earlier for the velocity. Thus, qualitatively, the influence of these three parameters and time on temperature is similar to that on velocity.

As regards the influence of the parameters Le, $n_{b}$, and $n_{t}$ on the nanoparticle volume fraction $\phi$, one may note that the Brownian motion parameter has negligible effect on it (Figure 9), while the Lewis number and the thermophoresis parameter have both a decreasing effect (Figures 8 and 10). The profiles of $\phi$ with respect to time $t$ are monotonic and attain steady-state with increasing time. This steady-state phenomenon is in contrast to what has been stated above for the nanofluid velocity and temperature.

3.1. Skin Friction, Surface Temperature, and Sherwood Number. In what follows, we will now introduce three specific quantities that are of practical interest for the convective flow considered here. These are the skin friction $\tau$, surface temperature $T(0, t)$, and the Sherwood number Sh at the left channel wall. The skin friction and the Sherwood numbers are defined, respectively, by the absolute values of the first derivatives of $u$ and $\phi$ with respect to the space variable $y$, evaluated at the wall $y=0$. In order to assess the influence of the four governing parameters on these quantities, we have presented a set of computed values in Table 1. From the table, we observe the following features.

(a) The thermophoresis parameter tends to enhance the wall shear stress as well as the surface temperature, for all times. However, the Sherwood number exhibits nonmonotonic behavior with increasing thermophoresis parameter.

(b) The effect of Brownian motion parameter is to increase all three quantities $\tau, T(0, t)$, and Sh. In terms of percentage increase, the shear stress is more sensitive to this parameter than the other two quantities. Within the transient time scales chosen, wall shear stress was seen to increase about 30 to 40 percent.

(c) The Lewis number tends to enhance all three quantities. However, the percentage change in this case is more pronounced for the Sherwood number. The percentage increase of this quantity was in the range of about 12 to 30 .

(d) During the early part of the transient stage, the wall shear stress and the surface temperature increase with time while the Sherwood number decreases. The shear stress increases approximately 400 percent while the surface temperature increases nearly 200 percent. On the other hand, the Sherwood number decreases sharply by over 80 percent.

(e) Except the Lewis number, the other parameters do not influence the steady-state (SS) time of these quantities.

\section{Conclusions}

We have studied numerically using an implicit finitedifference scheme the transient free convection in a vertical channel saturated by a nanofluid. The model incorporates 
the effects of Brownian diffusion and thermophoretic diffusion of the nanoparticles. We have analyzed the effects of these processes and the application of different types of thermal conditions-uniform heat flux and temperature at opposing walls. It is seen that the nanofluid velocity and temperature increase with the Brownian motion and thermophoresis parameters, but the Lewis number has a negligible effect on them. Significant changes occur in the Sherwood number in a small time interval immediately after the start of the motion. However, such a phenomenon does not occur in the case of skin friction. The model analyzed enables one to assess the relative influence of different types of thermal conditions at the channel walls and the main nanoparticle diffusion processes on the flow and heat transfer. The enhanced thermal property of the nanofluid observed in the study is of great interest in the thermal management of systems in several applications in engineering and medicine.

\section{Abbreviations}

\section{Nomenclature}

$D_{B}$ : Brownian diffusion

$D_{T}$ : Thermophoresis diffusion

g: Acceleration due to gravity

$k$ : Thermal conductivity of the nanofluid

$L$ : Perpendicular distance between the walls

Le: Lewis number

$N$ : Buoyancy ratio parameter

$n_{b}$ : Brownian motion parameter

$n_{t}$ : Thermophoresis parameter

Pr: Prandtl number

$q$ : Constant heat flux on the left wall

Sh: Sherwood number

$t$ : Nondimensional time

$t^{\prime}$ : Time variable

$T_{c}^{\prime}$ : Constant temperature of the right wall

$T^{\prime}$ : Temperature of the nanofluid

$T$ : Nondimensional temperature of the nanofluid

$u^{\prime}$ : Velocity of the nanofluid in the $x^{\prime}$ direction

$u$ : Nondimensional nanofluid velocity

$x^{\prime}$ : Co-ordinate along the left wall

$x$ : Nondimensional co-ordinate along the wall

$y^{\prime}$ : Co-ordinate perpendicular to the left wall

$y$ : Nondimensional co-ordinate perpendicular to the left wall.

\section{Greek Symbols}

$\alpha: \quad k /(\rho c)_{f}$

$\beta$ : Coefficient of thermal expansion

$\rho_{f}$ : Density of the nanofluid $\rho_{p}: \quad$ Density of the nanoparticles

$(\rho c)_{f}$ : Heat capacity of the fluid

$(\rho c)_{p}:$ Effective heat capacity of the particles

$\nu$ : Kinematic viscosity of the nanofluid

$\phi$ : Nanoparticle volume fraction in nondimensional form

$\phi^{\prime}$ : Nanoparticle volume fraction

$\phi_{c}^{\prime}$ : Volume fraction of nanoparticles at the right wall

$\phi_{h}^{\prime}$ : $\quad$ Volume fraction of nanoparticles at the left wall

$\tau: \quad$ Nondimensional skin friction at the left wall

$\mu$ : $\quad$ Viscosity of nanofluid.

\section{Conflict of Interests}

The authors declare that there is no conflict of interests regarding the publication of this paper.

\section{References}

[1] B. Gebhart, Y. Jaluria, R. L. Mahajan, and B. Sammakia, Buoyancy-Induced Flows and Transport, Hemisphere, Cambridge, UK, 1988.

[2] D. J. Nelson and B. D. Wood, "Combined heat and mass transfer natural convection between vertical parallel plates," International Journal of Heat and Mass Transfer, vol. 32, no. 9, pp. 1779-1787, 1989.

[3] A. K. Singh, H. R. Gholami, and V. M. Soundalgekar, "Transient free convection flow between two vertical parallel plates," Heat and Mass Transfer, vol. 31, no. 5, pp. 329-331, 1996.

[4] H. Masoda, A. Ebata, K. Teramae, and N. Hishinuma, "Alternation of thermal conductivity and viscosity of liquid by dispersing ultra-fine particles," Netsu Bussei, vol. 7, no. 4, pp. 227-223, 1993.

[5] S. Choi, "Enhancing thermal conductivity of fluids with nanoparticles," in Developments and Applications of NonNewtonian Flows: FED 231/MD 66, D. A. Siginer and H. P. Wang, Eds., pp. 99-105, ASME, New York, NY, USA, 1995.

[6] J. A. Eastman, S. U. S. Choi, S. Li, W. Yu, and L. J. Thompson, "Anomalously increased effective thermal conductivities of ethylene glycol-based nanofluids containing copper nanoparticles," Applied Physics Letters, vol. 78, no. 6, pp. 718-720, 2001.

[7] S. K. Das, N. Putra, P. Thiesen, and W. Roetzel, “Temperature dependence of thermal conductivity enhancement for nanofluids," Journal of Heat Transfer, vol. 125, no. 4, pp. 567-574, 2003.

[8] J. Buongiorno, "Convective transport in nanofluids," Journal of Heat Transfer, vol. 128, no. 3, pp. 240-250, 2006.

[9] K. Khanafer, K. Vafai, and M. Lightstone, "Buoyancy-driven heat transfer enhancement in a two-dimensional enclosure utilizing nanofluids," International Journal of Heat and Mass Transfer, vol. 46, no. 19, pp. 3639-3653, 2003.

[10] N. Putra, W. Roetzel, and S. K. Das, "Natural convection of nano-fluids," Heat and Mass Transfer, vol. 39, no. 8-9, pp. 775784, 2003.

[11] V. Trisaksri and S. Wongwises, "Critical review of heat transfer characteristics of nanofluids," Renewable and Sustainable Energy Reviews, vol. 11, no. 3, pp. 512-523, 2007. 
[12] X.-Q. Wang and A. S. Mujumdar, "Heat transfer characteristics of nanofluids: a review," International Journal of Thermal Sciences, vol. 46, no. 1, pp. 1-19, 2007.

[13] D. Y. Tzou, "Instability of nanofluids in natural convection," Journal of Heat Transfer, vol. 130, no. 7, Article ID 072401, 9 pages, 2008.

[14] D. A. Nield and A. V. Kuznetsov, "The Cheng-Minkowycz problem for natural convective boundary-layer flow in a porous medium saturated by a nanofluid," International Journal of Heat and Mass Transfer, vol. 52, no. 25-26, pp. 5792-5795, 2009.

[15] D. A. Nield and A. V. Kuznetsov, "Thermal instability in a porous medium layer saturated by a nanofluid," International Journal of Heat and Mass Transfer, vol. 52, no. 25-26, pp. 57965801, 2009.

[16] E. Abu-Nada and A. J. Chamkha, "Effect of nanofluid variable properties on natural convection in enclosures filled with a CuO-EG-water nanofluid," International Journal of Thermal Sciences, vol. 49, no. 12, pp. 2339-2352, 2010.

[17] A. Chamkha, R. S. R. Gorla, and K. Ghodeswar, "Nonsimilar solution for natural convective boundary layer flow over a sphere embedded in a porous medium saturated with a nanofluid," Transport in Porous Media, vol. 86, no. 1, pp. 13-22, 2011.

[18] R. S. R. Gorla and A. Chamkha, "Natural convective boundary layer flow over a nonisothermal vertical plate embedded in a porous medium saturated with a nanofluid," Nanoscale and Microscale Thermophysical Engineering, vol. 15, no. 2, pp. 81-94, 2011.

[19] A. J. Chamkha, S. Abbasbandy, A. M. Rashad, and K. Vajravelu, "Radiation effects on mixed convection over a wedge embedded in a porous medium filled with a nanofluid," Transport in Porous Media, vol. 91, no. 1, pp. 261-279, 2012.

[20] Z. Haddad, E. Abu-Nada, H. F. Oztop, and A. Mataoui, "Natural convection in nanofluids: are the thermophoresis and Brownian motion effects significant in nanofluid heat transfer enhancement?" International Journal of Thermal Sciences, vol. 57, pp. 152-162, 2012.

[21] N. C. Sacheti, P. Chandran, A. K. Singh, and B. S. Bhadauria, "Transient free convective flow of a nanofluid in a vertical channel," International Journal of Energy \& Technology, vol. 4, pp. 1-7, 2012. 


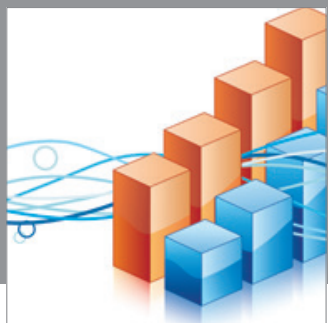

Advances in

Operations Research

mansans

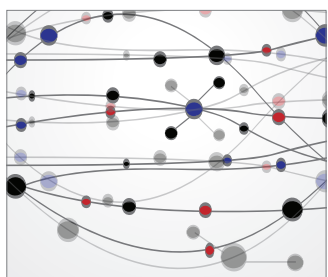

The Scientific World Journal
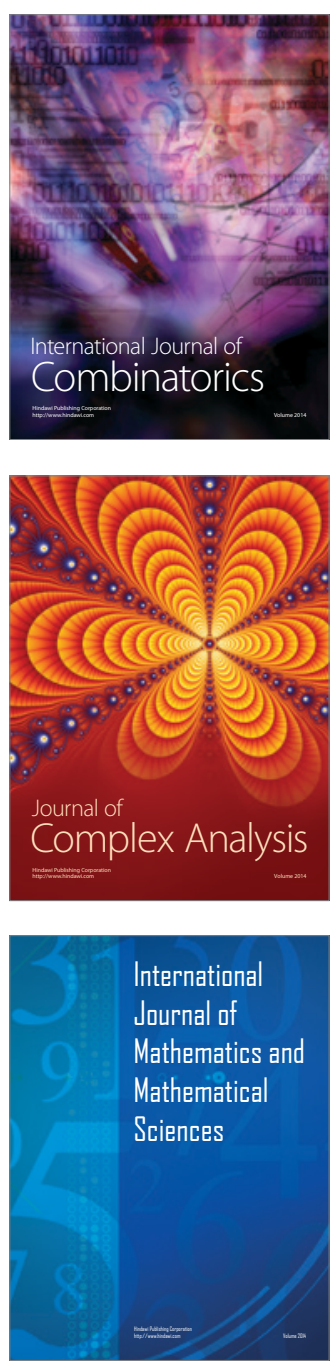
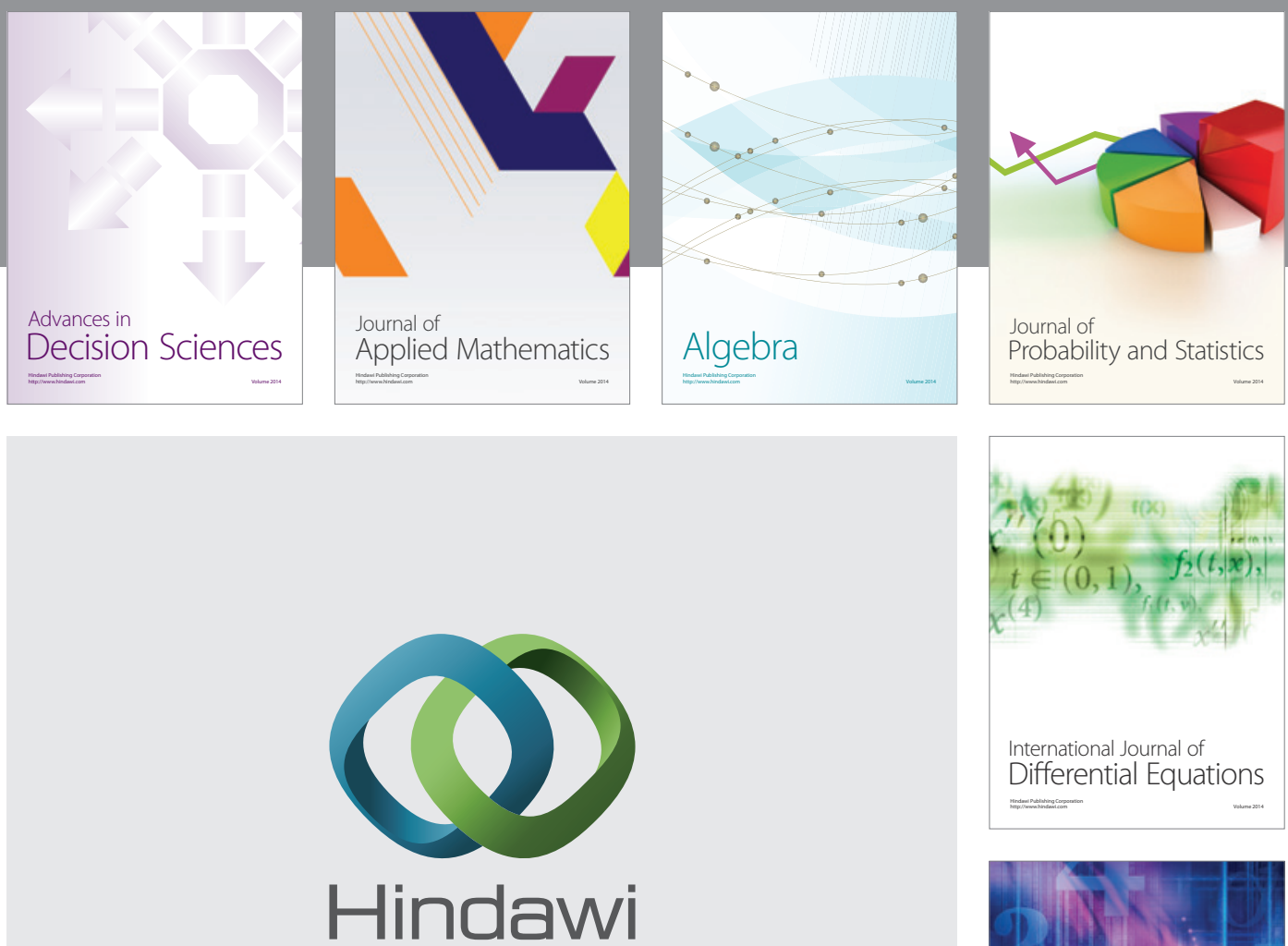

Submit your manuscripts at http://www.hindawi.com
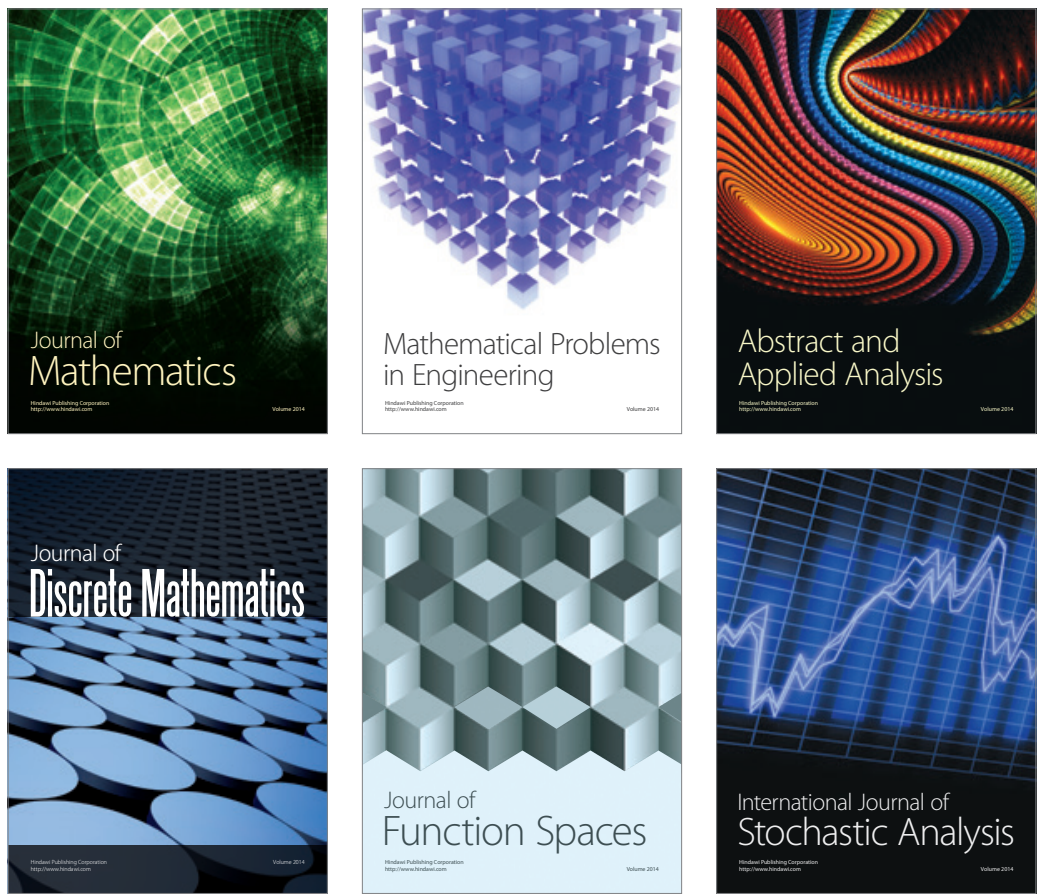

Journal of

Function Spaces

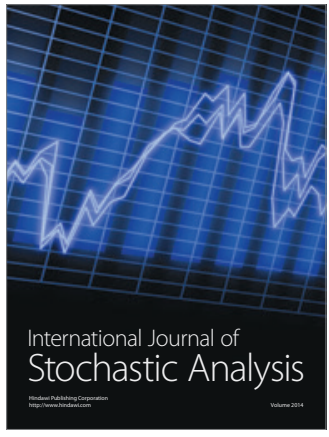

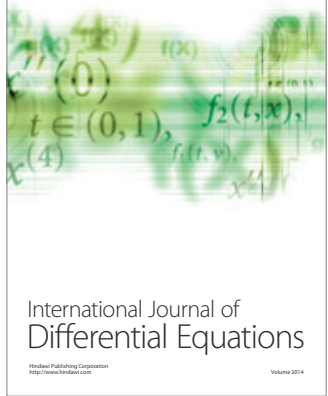
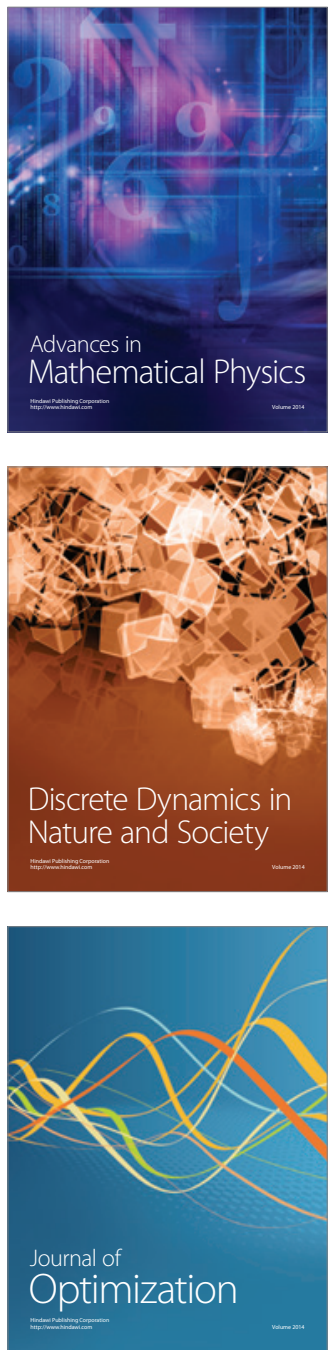